\title{
Desarrollo de nueva metodología de diseño, análisis y aplicaciones de Micro y Nanoinstrumentación inteligente soportadas en Ingeniería en Nano- biotecnología para la Automatización Industrial
}

\section{Development of new methodology of design, analysis and applications of intelligent Micro and Nano- instrumentation supported in Nanobiotechnology Engineering for Industrial Automation}

DOI: http://doi.org/10.17981/ingecuc.15.2.2019.12

Artículo de Investigación Científica. Fecha de Recepción: 05/04/2019. Fecha de Aceptación:.6/11/2019

\author{
Antonio Faustino Muñoz-Moner \\ Universidad Autónoma de Bucaramanga. Bucaramanga (Colombia) \\ amunozm@unab.edu.co \\ Aldo Pardo-García \\ Universidad de Pamplona. Pamplona (Colombia) \\ apardo13@unipamplona.edu.co \\ Pedro Miguel Caicedo-Torres \\ Universidad Autónoma de Bucaramanga. Bucaramanga (Colombia) \\ pcaicedo@unab.edu.co
}

Para citar este artículo:

A. Muñoz-Moner, A. Pardo Pardo-García y P. Caicedo-Torres. "Desarrollo de nueva metodología de diseño, análisis y aplicaciones de micro y nanoinstrumentación inteligente soportadas en Ingeniería de Nanobiotecnología para la Automatización Industrial”, INGE CUC, vol. 15, no. 2, pp. 123-131, 2019. DOI: http://doi.org/10.17981/ingecuc.15.2.2019.12

\section{Resumen}

Introducción- El presente artículo muestra los resultados de la investigación realizada en el proyecto sobre diseño y desarrollo de nuevas metodologías para el diseño, análisis y aplicación de micro y nanoinstrumentación inteligente soportada en Ingeniería en Nanobiotecnología por tecnología de monitoreo en tiempo real del contenido de fenoles en las aguas residuales industriales, flujos de vertimiento en línea de ductos, tanques de tratamiento, piscinas de estabilización a cielo abierto y en la descarga del vertimiento del agua como parte del desafío presentado por Ecopetrol sobre la necesidad de descontaminación de las aguas residuales en la Refinería de Petróleo de Barrancabermeja.

Objetivo- Lo propuesto anteriormente se traduce en una red de sensores que garantiza la redundancia necesaria para la confiabilidad técnica, que incluye un Sistema Instrumentado de Seguridad (SIS) dotado de un Sistema de Control Redundante (RCS), lo que asegura el funcionamiento óptimo de la instrumentación implementada y además permite reducir el tiempo de medición en cada secuencia de muestreo.

Metodología- A través de la detección de contaminantes en tiempo real se utiliza una red de sensores basados en micro y_nanobioinstrumetación con nariz electrónica, lengua artificial y ojo espectrofotométrico, soportada en tecnología móvil para el monitoreo de los parámetros de calidad del agua (contenido de fenoles) en líneas de tubería en vertimiento y afluentes.

Resultados- En el articulo se exponen los resultados desarrollados de un sistema de monitoreo en tiempo real y control on-line por tecnología móvil de parámetros de calidad del agua (fenoles) en línea de tuberías para vertimientos y afluentes emulados por la replicación funcional del funcionamiento de los sentidos del olfato, sabor y de la visión espectrofotométrica por clonación artificial aplicados en el diseño de una red de sensores y sistemas de control.

Conclusiones- En síntesis, el proyecto presenta una solución alternativa viable soportada en indicadores de alto impacto, técnico-tecnológico, económico y confiable, donde la información se presenta en tiempo real con un registro cada dos minutos, y acceso omnipresente (desde cualquier lugar) por tecnología móvil, con una disminución de los costos de fabricación implementación en más de cien veces por utilización de MEMS y NEMS.

Palabras clave- Red de sensores; concentración de fenoles; micro-bioinstrumentación; nano-bioinstrumentación

\section{Abstract}

Introduction- The present article shows the results of the research carried out in the project on design and development of new methodologies for the design, analysis and application of intelligent micro and nano instrumentation supported in Nanobiotechnology Engineering by real time monitoring technology of the phenol content in industrial wastewater, duct line discharge flows, treatment tanks, open-air stabilization pools and water discharge as part of the challenge presented by Ecopetrol on the need to decontaminate waste water at the Barrancabermeja Oil Refinery.

Objective- The above proposal translates into a sensor network that guarantees the necessary redundancy for technical reliability, which includes a Safety Instrumented System (SIS) equipped with a Redundant Control System (RCS), which ensures optimal operation of the instrumentation implemented and also allows for a reduction in measurement time in each sampling sequence.

Methodology- Through the detection of contaminants in real time, a network of sensors based on micro y_nanobioinstrumentation with electronic nose, artificial tongue and spectrophotometric eye is used, supported by mobile technology for monitoring water quality parameters (phenol content) in discharge and affluent pipelines.

Results- The article presents the results developed from a real-time monitoring system and on-line control by mobile technology of water quality parameters (phenols) in pipelines for discharges and tributaries emulated by the functional replication of the functioning of the senses of smell, taste and spectrophotometric vision by artificial cloning applied in the design of a network of sensors and control systems.

Conclusions- In summary, the project presents a viable alternative solution supported by high impact, technical-technological, economic and reliable indicators, where the information is presented in real time with a record every two minutes, and omnipresent access (from anywhere) by mobile technology, with a decrease in manufacturing and implementation costs by more than one hundred times through the use of MEMS and NEMS.

Keywords-Sensor network; phenol concentration; micro-bioinstrumentation; nano-bioinstrumentation 


\section{INTRODUCCIÓN}

En el proyecto se lleva a cabo con el diseño, desarrollo e implementación de un dispositivo de pruebas de electrohilado (electrospinning ) para aplicaciones en nanoinstrumentación que controla las variables físicas que intervienen en la producción de nanohilos como aporte en sus proyectos del nuevo programa de pregrado de Ingeniería Biomédica, el Proyecto FOSUNAB, Proyectos del Doctorado en Ingeniería Red Mutis, de la Maestría en Automatización Industrial y Mecatrónica los Programas de Ingeniería Mecatrónica e Ingeniería de Sistemas, los resultados contribuyen con nuevos conocimientos para la electiva de profundización en Aplicación de Sistemas nanotecnológicos en Ingeniería, para las estudiantes de ingeniería.

Lo propuesto anteriormente se traduce en una red de sensores que garantiza la redundancia necesaria para la confiabilidad técnica, que incluye un Sistema Instrumentado de Seguridad (SIS) dotado de un Sistema de Control Redundante (RCS), lo que asegura el funcionamiento óptimo de la instrumentación implementada además permite reducir el tiempo de medición en cada secuencia de muestreo a 2 minutos junto con una mayor duración de la instrumentación compuesta por micromateriales de alta resistencia y duración con un bajo mantenimiento gracias al alto grado de compactación utilizado.

\section{Desarrollo}

La implementación de obleas de NEMS (Nano-Electromechanicals Systems) y MEMS (Micro-Electro- mechanicals Systems) con multisensores se logra alcanzar un costo competitivo y de gran facilidad para su fabricación, además de obtener un alargamiento de la vida útil de la instrumentación, mayor seguridad para el personal y el proceso y un mejoramiento de la fiabilidad del sistema con el SIS que utiliza RCS. En la Fig.1 se muestran las diferentes etapas correspondientes al dimensionamiento de este modelo de sensores a nanoescala.

Para la Nariz electrónica el método de diseño se basa en el desarrollo de una red de sensores fundamentados en la medición de olor de las aguas residuales con nariz electrónica, por interacción de electrodos con el gas que produce un cambio en la resistividad del material semiconductor con óxidos metálicos, complementado con nanopartículas de nanotubos de carbono y/o polímeros conductores. Para la de lengua artificial se utiliza el método de medición soportado en sensores electroquímicos amperométricos que determinan la reacción de oxidación/reducción de sustancias electroactivas, presentes en la disolución de agua (fenoles), aplicando un potencial determinado y registrando la intensidad de la corriente resultante que es una medida directa de la concentración del analíto presente en la disolución. Los cambios en la constitución de los electrodos permite garantizar su selectividad y los valores de impedancia electroquímica, para detectar la adsorción de sustancias en la superficie del electrodo, recubierta con materiales como polímeros conductores, nanotubos de carbono o ftalocianinas con diferentes potenciales. Y para el ojo espectrofotométrico (Fig. 2) se considera el método basado

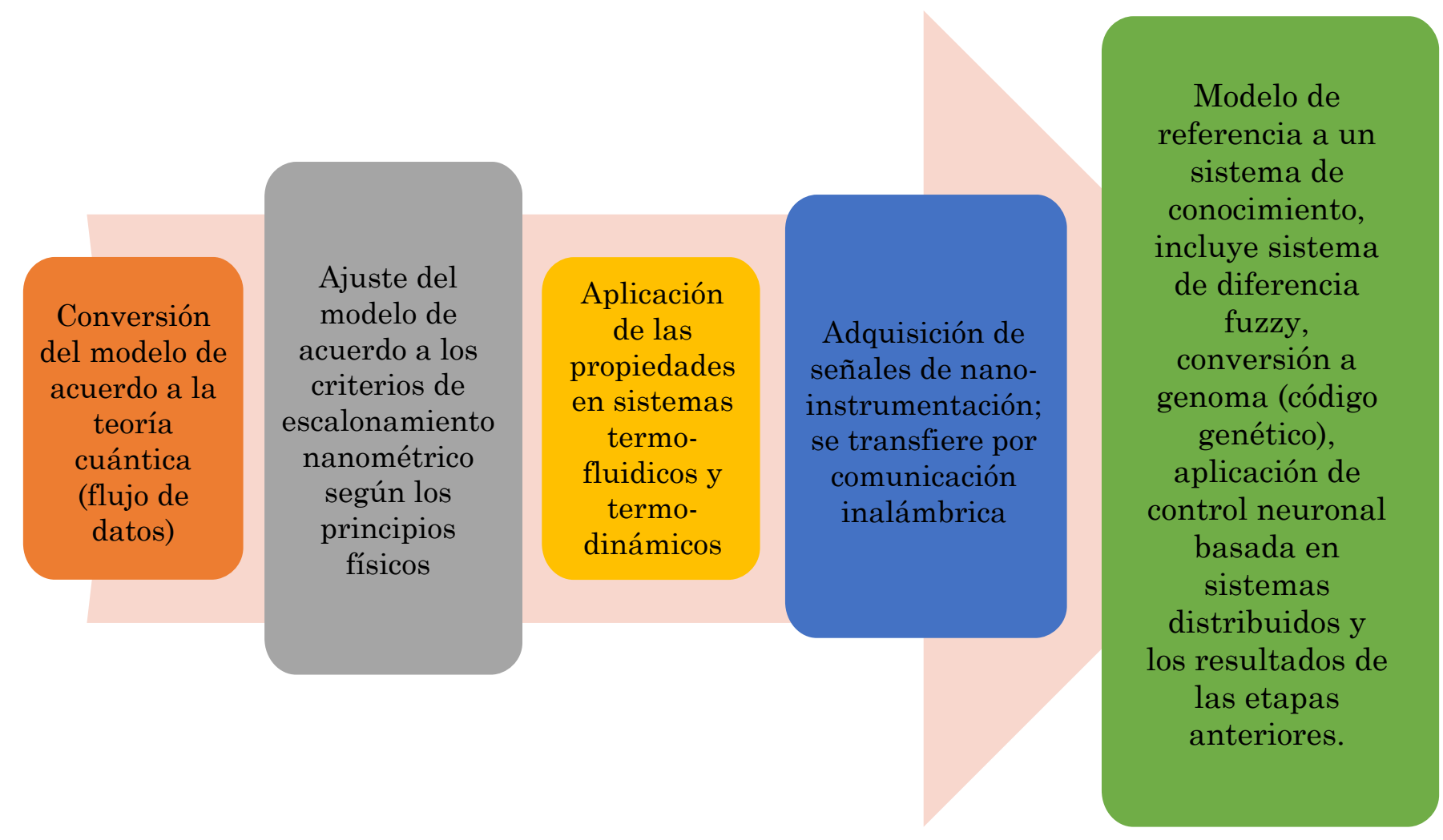

Fig. 1. Modelo de medicion a nanoescala. Fuente: Autores. 
en visión por emisión plasma que consiste en la obtención de una llama de elevada temperatura que transforma la mezcla de combustible a estado de plasma lo que a partir de una mezcla de gases se determina la concentración de cationes y electrones lo que permite una elevada eficiencia en la atomización y la emisión de los átomos y la determinación de la cantidad de elementos químicos contaminantes (fenoles) con alta sensibilidad y precisión; el sistema de red sensores |implementado por tecnología móvil conforman el sistema monitoreo en tiempo real de la concentración de contaminantes y determinación de la calidad del agua (fenoles) en línea de tuberías para vertimiento y en efluentes. Con la interacción gas-sensor que produce un cambio en la resistividad del material sensible, los sensores más utilizados en narices electrónicas están basados en sensores resistivos y dentro de esta categoría los más utilizados son los sensores que utilizan materiales con nanotubos de carbono y polímeros conductores [1][5], polímeros conductores [7].

En la Lengua Electrónica las medidas de impedancia electroquímica, con la que se puede de- tectar la adsorción de sustancias a la superficie del electrodo a diferentes potenciales. Las lenguas electrónicas basadas en espectroscopía de impedancias constan de una red de electrodos recubiertos de diferentes materiales sensibles (polímeros conductores, nanotubos de carbono o ftalocianinas entre otros [4]-[8]. La Lengua Electrónica basada en sensores electroquímicos amperométricos por medición y valoración de oxidación/reducción de especies electroactivas presentes en la disolución agua (fenoles), cuando se aplica un cierto potencial; la corriente registrada $(\mu \mathrm{A})$ es una medida directa de la concentración del analito presente en la disolución. La modificación de los electrodos permite obtener redes de sensores con diferente selectividad y/o medidas de impedancia electroquímica, con la que se puede detectar la adsorción de sustancias a la superficie del electrodo a diferentes potenciales. Las lenguas electrónicas basadas en espectroscopía de impedancias constan de una red de electrodos recubiertos de diferentes materiales sensibles (polímeros conductores, nanotubos de carbono o ftalocianinas entre otros).

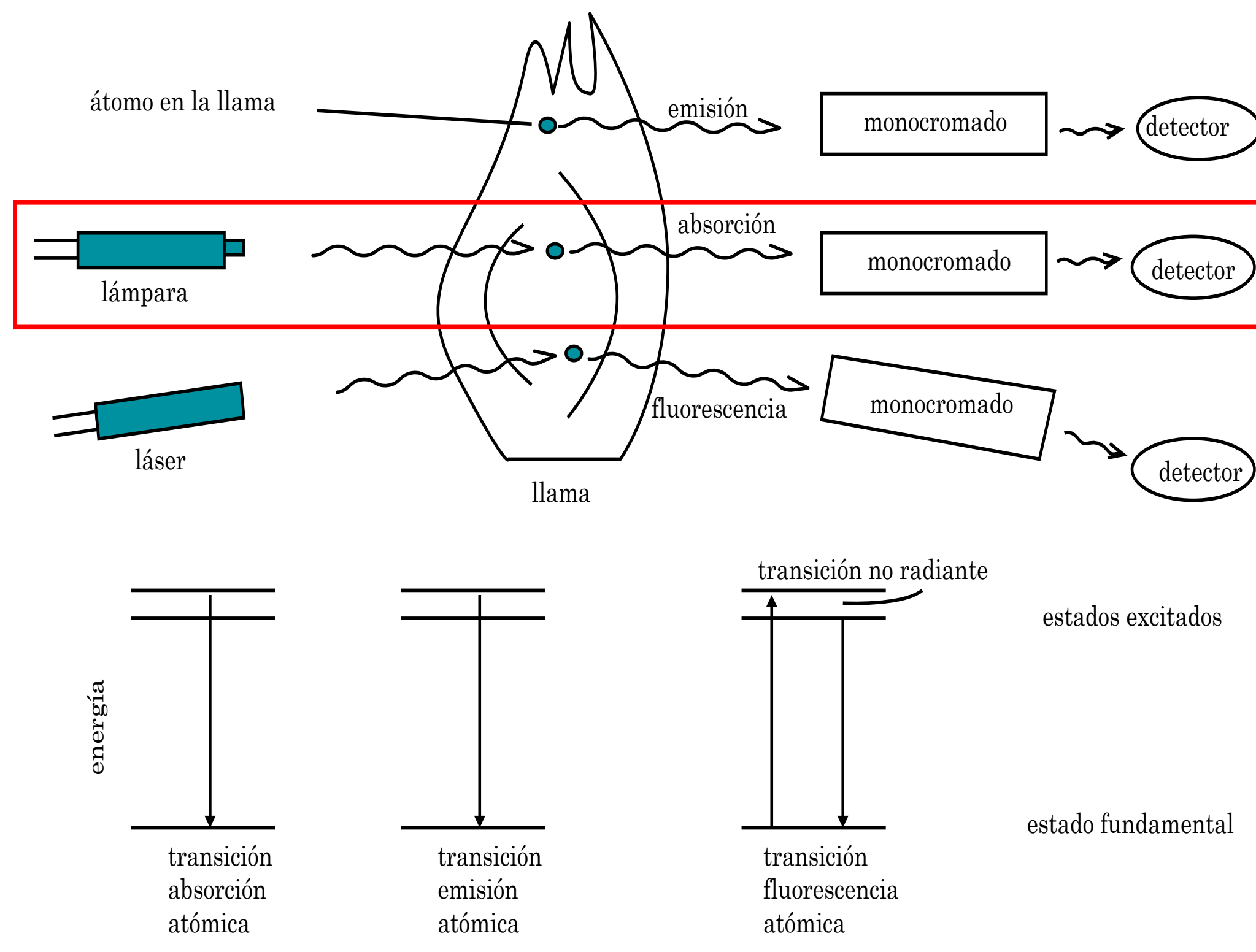

Fig. 2. Ojo espectrofotométrico. Fuente: Autores. 


\section{Resultados}

El desarrollo de nanosensores por fabricación basados en la técnica del electrohilado para implementación de nanoinstrumentación a partir de nanohilos considera la variación de su resistencia eléctrica a partir de la relación conocida de la resistencia del conductor (1).

$d R=\frac{\partial R}{\partial \rho} \cdot d \rho+\frac{\partial R}{\partial L} \cdot d L+\frac{\partial R}{\partial A} \cdot d A ;$

Donde,

$\frac{\partial R}{\partial \rho}=L / A ; \frac{\partial R}{\partial L}=\rho / A ; \frac{\partial R}{\partial A}=-\rho L / A^{2}$

Entonces,

$d R=\frac{A(p d L+L d p)-p L d A}{A^{2}}$

La sección transversal se puede reducir a una sección circular de diámetro equivalente, y por lo tanto:

$A=Q d_{e}{ }^{2}$

El conductor es circular, $Q=\pi / 4$.

Derivando,

$d A=2 Q d_{e} d\left(d_{e}\right)$

Recordando la magnitud del coeficiente de Poisson:

$\frac{d\left(d_{e}\right)}{d_{e}}=-\mu \frac{d L}{L}$

Resulta entonces (7):

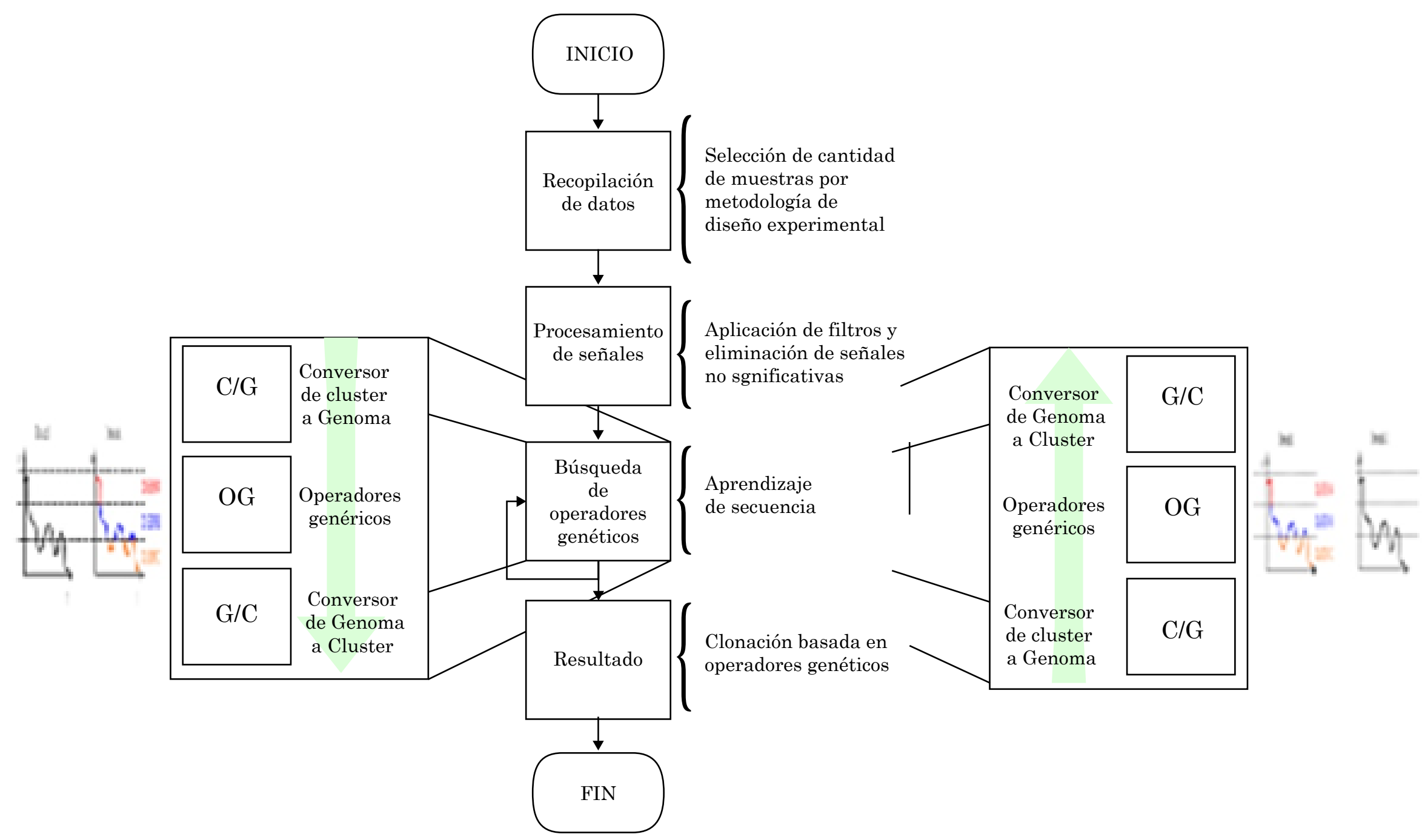

Fig. 3. Diagrama de la metodología aplicada.

Fuente: Autores. $d A=-2 Q d_{e}^{2} \mu \frac{d L}{L}=-2 A \mu \frac{d L}{L}$

Sustituyendo la ecuación (9) en la ecuación (8).

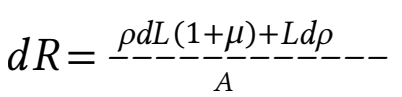

Eliminando $A$ con la ayuda de la ecuación (7).

$\frac{d R}{d}=\left(1+2 \mu+\frac{d \rho / \rho}{d L / L}\right) \frac{d L}{L}$

Ahora se toma en cuenta que la deformación longitudinal unitaria $\varepsilon=\Delta L / L$ y entonces:

$\frac{d R}{d}=K_{g} \varepsilon ; K_{g}=1+2 \mu+\frac{d \rho / \rho}{\varepsilon}$

Se observa que $K g$ depende aparentemente de $\varepsilon$ pero el término $(d p / p)$ es una propiedad física del material del conductor denominado piezorresistividad, de forma:

$\frac{d \rho / \rho}{\varepsilon}=\pi_{1} E$

En la ecuación (13), $E$ es el modulo de la elasticidad del material y $\pi_{1}$ es el coeficiente de piezorresistividad longitudinal, pudiendo ser positivo o negativo. Finalmente,

$\frac{d R}{d}=K_{g} \varepsilon ; K_{g}=1+2 \mu+\pi_{1} \varepsilon$

$K_{g}$ es el coeficiente del tensoelemento y relaciona la deformación longitudinal unitaria (cambio relativo de longitud) con el cambio relativo de resistencia del conductor. 
La metodología del diseño aplicada en la conceptualización por replicación funciona y clonación artificial en ingeniería.es una alternativa para el desarrollo de medios y sistemas de control avanzado, que permitan responder a las exigencias de la industria, a través de réplicas funcionales de sensores, controladores y actuadores basados en técnicas de inteligencia artificial soportados en sistemas avanzados de clonación artificial y software evolutivo (Fig. 3).

La metodología de clonación artificial en ingeniería parte de un sistema basado en conocimiento; se fundamenta en la teoría de los sistemas expertos; puede partir de información cualitativa, pero igual la base de conocimiento puede estar generada por información de sensores sobre variables físicas del proceso en cuyo caso las reglas son el resultado de la interacción de los datos de entrada-salida.

Para esta metodología se proponen:

1. Creación de los clusters.

2. Creación de los cromosomas.

3. Búsqueda de consecuentes.

4. Identificar los operadores correctos.

5. Crear algoritmo genético que busque la secuencia.

6. Evaluar los individuos.

7. Seleccionar los mejores (evaluados según error).

8. Reproducir y generar nueva población.

9. Repetir ciclo optimizando el error.

10. Identificación de valores de salida.

11. Desfuzzificación.

El conjunto de datos entregado por el proceso de clonación, es procesado por el sistema, una de las etapas importantes de este proceso es la defusificación de una parte del "cromosoma", de tal forma que podamos tener el valor de la variable clonada en rangos del universo de discurso y no en términos de pertenencia a conjuntos borrosos (Fig. 4).
El conjunto de datos entregado por el proceso de clonación, es procesado por el sistema, una de las etapas importantes de este proceso es la defuzzificación de una parte del "cromosoma", de tal forma que podamos tener el valor de la variable clonada en rangos del universo de discurso y no en términos de pertenencia a conjuntos borrosos.

Para la validación experimental de los resultados [9] de la utilización del equipo de electrohilado para la fabricación de los nanohilos se procede con el diseño experimental basada en tres series de expermientos para diferentes concentraciones de polimero consevido a travez de experimentos cuyo diseño corresponde al analisis de muestras y sus resultados a travez de espacios de superficies de respuesta; el procesamiento de los resultados de las muestras con fines de validación se estructura a traves de procedimiento de superposición de la imagen obtenida de las muestras y el calculo por ingenieria en nanotecnologia soportado en el modelo cuantico validado a traves del software SIESTA (WinWulff SIESTA) [20].

El diseño experimental basado en superficie de respuesta considera los factores que intervien en la obtencion de las muestras incluyendo la estimación de los modelos cuanticos a traves de los graficos de contorno y los metos para el analisis de las superficies obtenidas. De acuerdo al diseño experimental de tres series de experimentos para diferentes concentraciones (6\%,8\% y $10 \%)$, para cada uno de estos se realizaron entre 5 y 7 pruebas para un total de 15 a 17 muestras, el analisis estadistico de las muestras obtenidas se puede observar mas adelante.

Las pruebas realizadas para tres concentraciones diferentes tuvieron en cuenta las variables de funcionamiento del equipo de electrohilado y se tomaron como factores significativos, el desplazamiento entre el inyector y colector y adicional a esto el voltaje de la

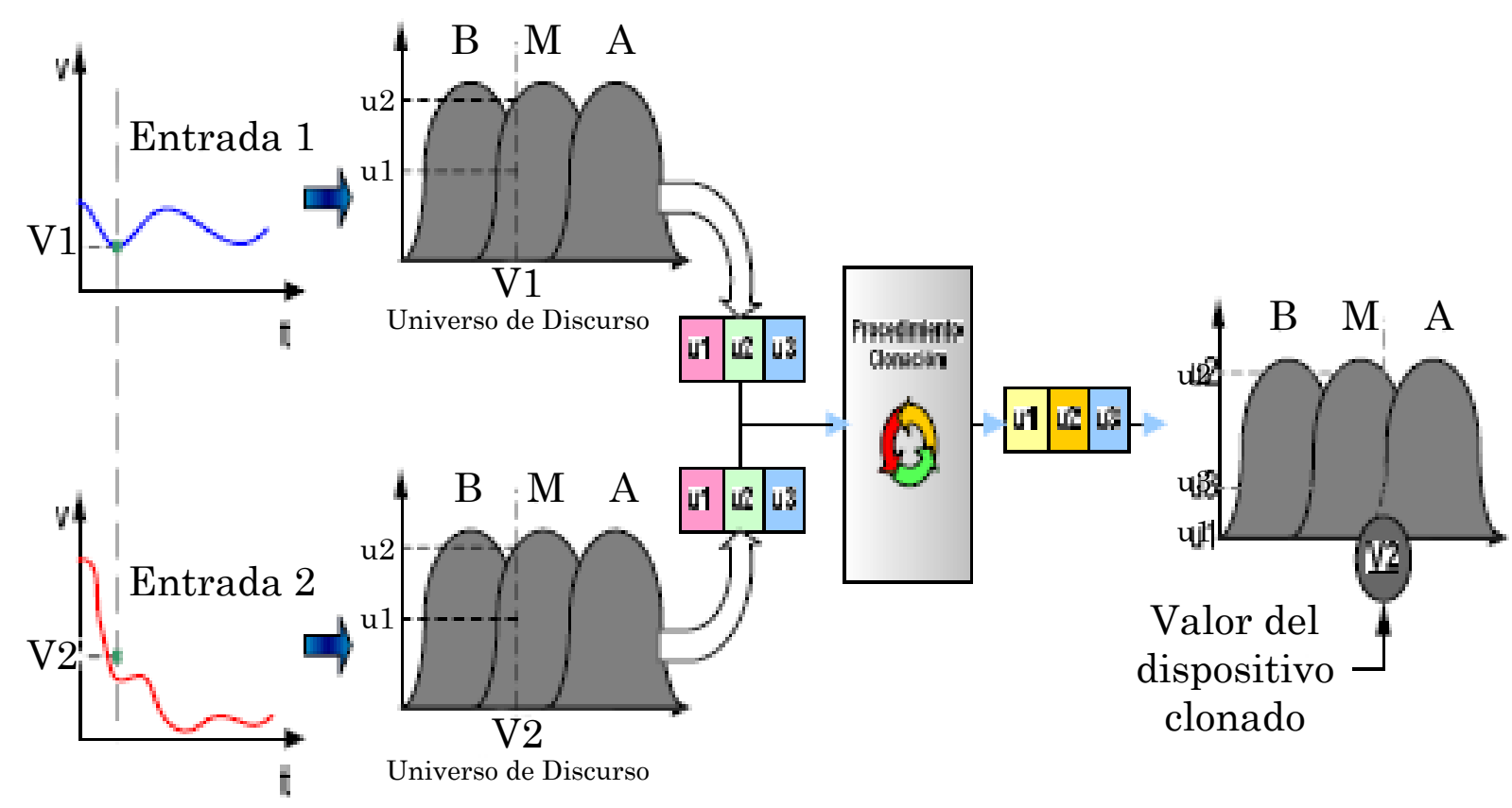

Fig. 4. Procedimientos de Clonación. Fuente: Autores. 
fuente de alimentación que garantiza el campo electrico de alto voltaje para la obtencion de nanohilos, desarrollado por la tecnología de monitoreo en tiempo real del contenido de fenoles en aguas residuales industriales, permitiendo conocer este parámetro en línea, ya sea en ductos, en tanques de tratamiento, en piscinas de estabilización a cielo abierto y/o directamente en la descarga del vertimiento, con un límite de detección de $0.1 \mathrm{mg} / \mathrm{L}$. Los datos obtenidos de las pruebas realizadas aparecen en las tablas y las imágenes de las muestras obtenidas a que se refieren las imágenes.
Para el procesamiento de los resultados de las pruebas realizads se realizaron calculos por superposición de imagen, donde la imagen original corresponde a la muestra obtenida con el equipo de electrohilado y la imagen super puesta corresponde a la modelacion obtenida con el software SIESTA (WinWulff SIESTA) [9]. Los resultados obtenidos del analisis de las muestras de polimero por superposición de acuerdo a las pruebas realizadas se muestran a continuación (Fig. 5, Fig. 6 y Fig. 7).

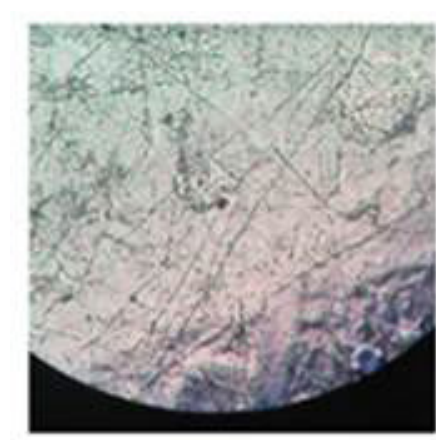

a)

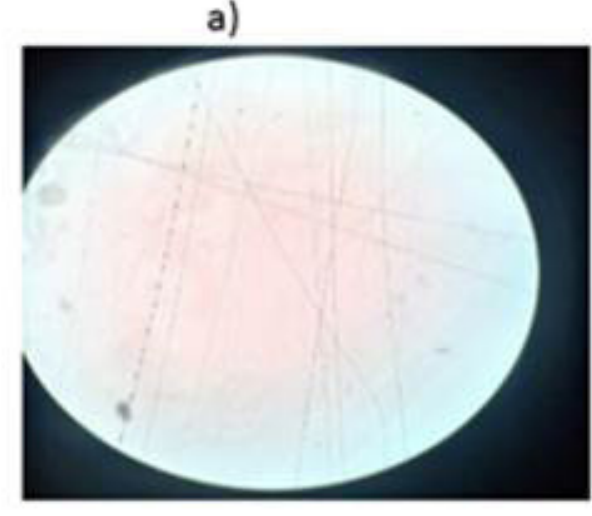

d)

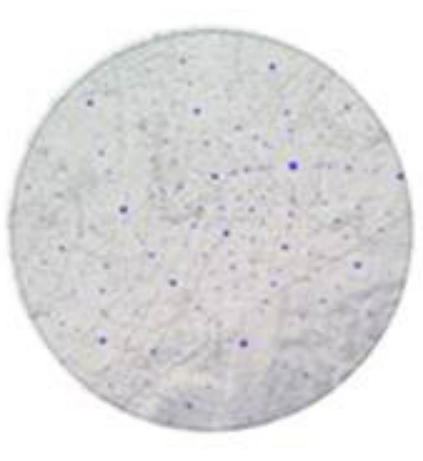

b)

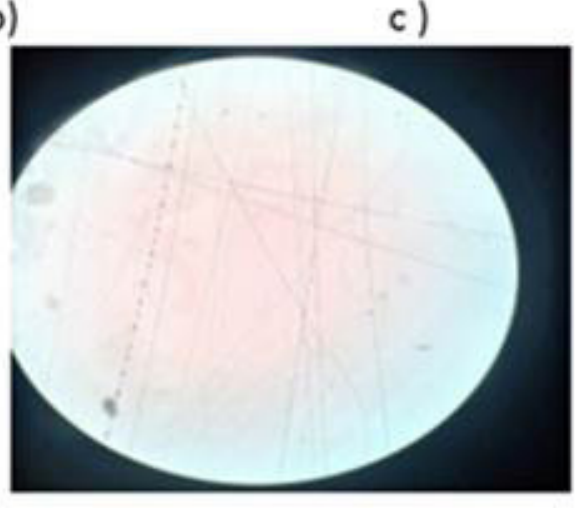

e)

Fig. 5. Muestras de nanohilos estructurados con 6\% de concentración del polímero. Fuente: Adaptado Autores.

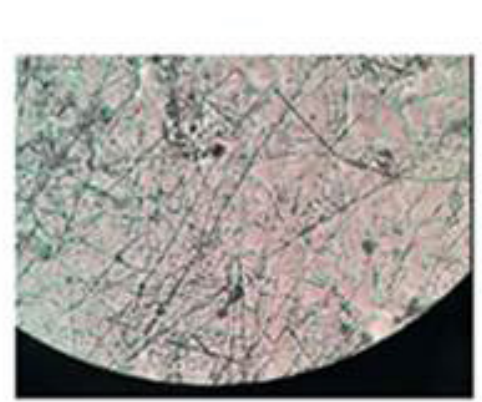

a)

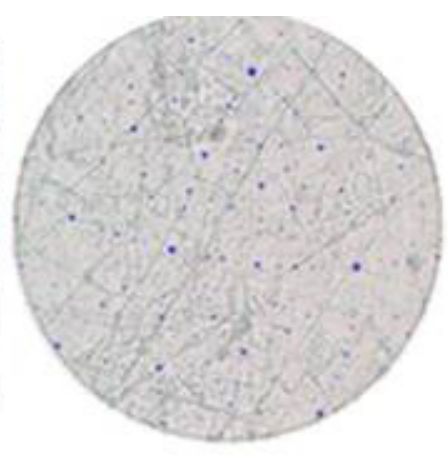

b)

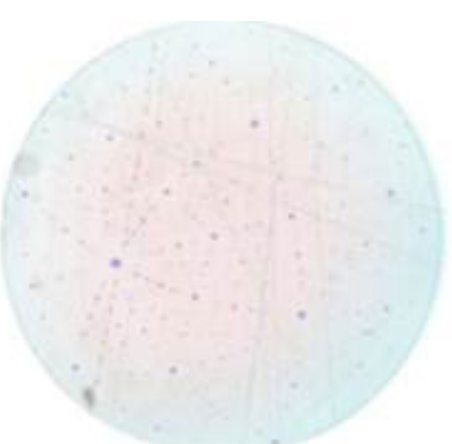

c)

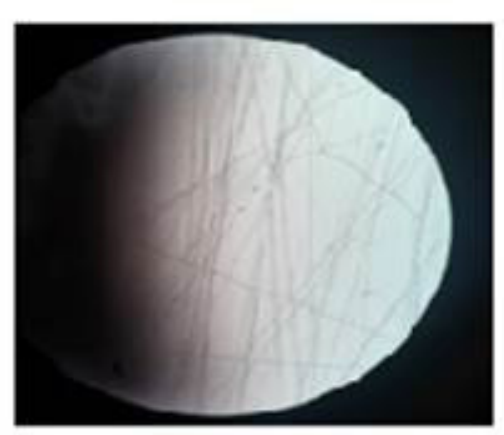

d)

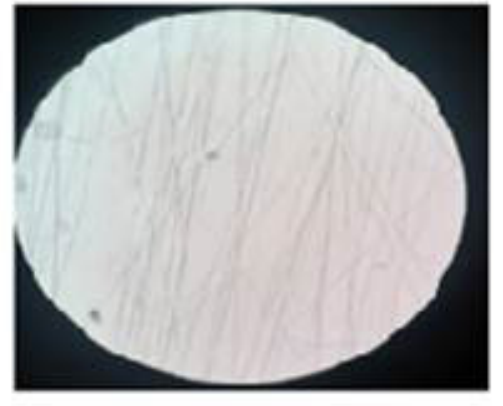

e)

Fig. 6. Muestras de nanohilos estructurados con $8 \%$ de concentración del polímero. Fuente: Autores. 


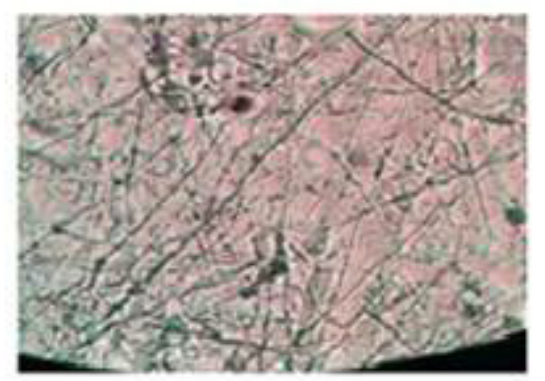

a)

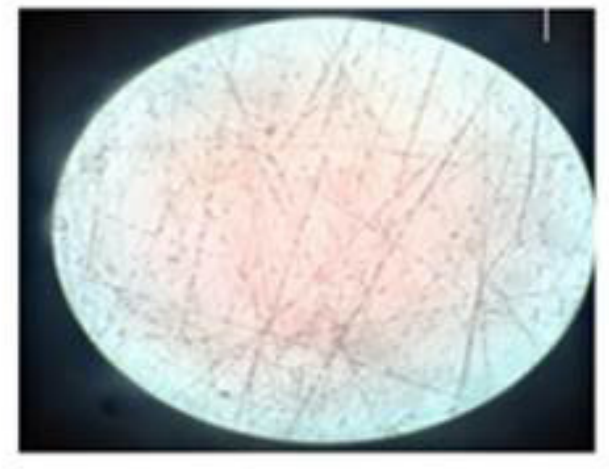

d)

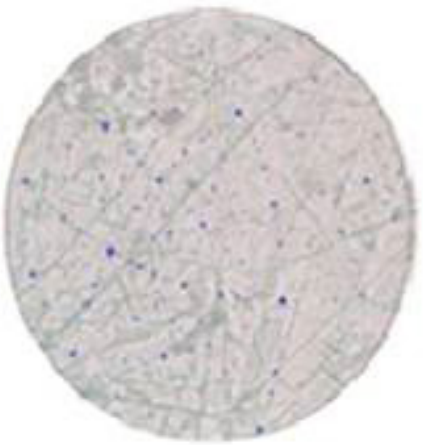

b)

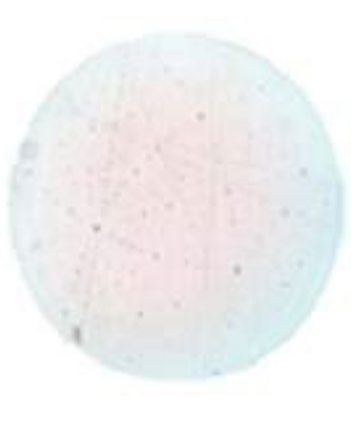

c)

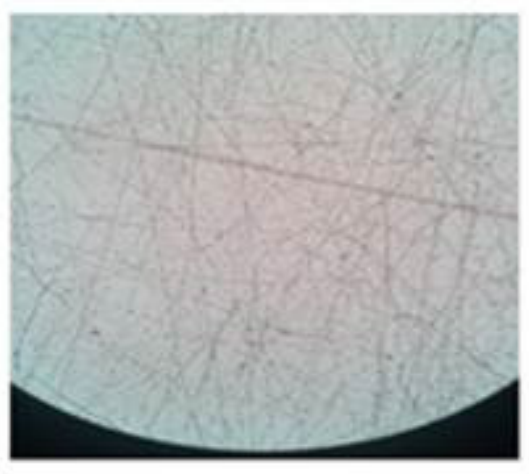

e)

Fig. 7. Muestras de nanohilos estructurados con $10 \%$ de concentración del polímero. Fuente: Autores.

Para la determinación de las propiedades electricas de las muestras obtenidas por electrohilado se aplica la norma ISO TC 213 correspondiente a la metodologia establecida por nanometrologia para aplicaciones en dispositivos a nanoescala [10]-[15]. A partir de los datos patrones establecidos por la norma para la validación de nanoestructuras con nanohilos establecidas como producto del proceso de hilado electronico (Norma de nanotecnologia sobre Caracterización eléctrica de fibras poliméricas obtenidas por electrohilado a través de las curvas I/V obtenidas por modelación y simulación por nanotecnología computacional para 1D en conductividad longitudinal).

\section{Conclusiones}

En síntesis el proyecto presenta una solución alternativa viable soportada en indicadores de alto impacto técnico-tecnológicos, económicos y confiables donde la información se presenta en tiempo real su registro es cada dos minutos, el acceso omnipresente (desde cualquier lugar) por tecnología móvil, con una disminución de los costos de fabricación e implementación en más de cien veces por utilización de MEMS y NEMS, el ahorro por disminución del mantenimiento de la red de sensores de medición e instrumentación del sistema de seguridad SIS en un $25 \%$, el alcance de la reducción de la contaminación con fenoles es del 97\%, la recuperación de la inversión y comercialización se alcanza en un plazo de 3 años y el registro de la patente en dos años; durante la implementación y el uso de la solución a la problemática de contaminación de las aguas en vertimientos y efluentes.
Además, la red de sensores emula el funcionamiento de los sentidos del olfato, gusto y visión y aplica la metodología avanzada de clonación artificial por replicación funcional en el diseño de la red de sensores y el sistema de control redundante, con técnicas de inteligencia artificial basada en algoritmos genéticos, redes neuronales artificiales y clusters fuzzy por circuitos lógicos FPGA's con hardware evolutivo que garantiza la adaptabilidad y flexibilidad de la aplicación de la red de sensores utilizada la concepción de la metodología Bottom up para la implementación de los sistemas adaptrónicos dotados de nanosensores en fusión de las tecnologías de sensores y las funciones de actuadores en los materiales inteligentes [16][19] ha dado como resultado una nueva tecnología: adaptrónica o materiales adaptables. La idea de esta nueva tecnología es utilizar materiales inteligentes que funcionen como sensores para que detecten -por ejemplo- cambios en el medio ambiente como presión, temperatura, humedad, y puedan responder a estos cambios de manera controlada por medio de actuadores; en adaptrónica, el sensor y el actuador se encuentran integrados en una misma estructura. El sensor detecta cambios en su entorno, y envía esta información al sistema de control, que produce una señal de respuesta y la enva al actuador.

Los aportes de los resultados obtenidos está soportada en que la solución incluye una Red de Sensores que garantiza la redundancia necesaria para garantizar la confiabilidad técnica de la propuesta de solución, un Sistema Instrumentado de Seguridad-SIS dotado de RCS (Sistema de Control Redundante) que asegura el 
funcionamiento óptimo de la instrumentación implementada en red de Sensores, la reducción del tiempo de medición en cada secuencia de muestreo a 2 minutos, una mayor duración de la instrumentación dotada de micro-nanomateriales de alta resistencia, alta duración, bajo mantenimiento, debido a la utilización de micro-nanobioinstrumentación de alto grado compactación, bajo costo referido a la utilización de obleas de NEMS (Nano Electromechanicals Systems) y MEMS (Micro-Electromechanicals Systems), con multisensores lo cual permite alcanzar un costo competitivo y gran facilidad para su fabricación, el alargamiento de la vida útil de la instrumentación soportada en NEMS y MEMS, una mayor seguridad para el personal y el proceso y mejora la fiabilidad del sistema con el SIS que utiliza RCS (Sistema de Control Redundante)

\section{Financiamiento}

Artículo de investigación científica derivado del proyecto de investigación en la convocatoria bienal titulado "Diseño y desarrollo del Laboratorio de Nanotecnología como parte del proyecto FOSUNAB" financiado por la UNAB que está orientado a continuar las investigaciones en Nanotecnología como parte de la prospectiva de los planes de desarrollo de la Facultad de Ingenierías Año de inicio: 2016, año de finalización: 2018

\section{Agradecimientos}

Agradecimiento especial al apoyo brindado por las instituciones y empresas involucradas en el proyecto ECOPETROL, IP-ECOPETROL, UNAB, ESSI Colombia, MOTUM DATA y reconocimiento al Semillero de Instrumentación y Control, que dispone del apoyo en alianza con el Laboratorio de Nanotecnología de la Universidad Técnica de Dinamarca y el Laboratorio de Biotecnología y Ambiente de la UNAB. Por el apoyo en la realización de pruebas para la fabricación de nanosensores con la ayuda de la dirección del Centro de Investigaciones de Dinamarca y la participación del Dr. Jaime Castillo León (investigador del Nano BioIntegrated System Group-NaBIS) y profesor de la misma universidad en el desarrollo de las primeras pruebas de fabricación de nanosensores en el centro de investigación y producción en nanotecnología del Technical University-DTU (Denmark).

\section{REFERENCIAS}

[1] G. Feng, "A Survey on Analysis and Design of ModelBased Fuzzy Control Systems", IEEE T. Fuzzy Syst., vol. 14, no. 5, pp. 676-697, Nov. 2006. https://doi.org/10.1109/ TFUZZ.2006.883415

[2] A. F. Muñoz \& K. A. Pardo, "The technology of advanced control of applied artificial cloning to highly precise mechatronical systems," in IEEE International Symposium on Intelligent Control, ISIC, Houston, TX, USA, Oct. 8, 2003, pp. 714-718. https://doi.org/10.1109/ISIC.2003.1254723
[3] S. F. A. Rahman, N. Azah, U. Hashim \& M. N. M. Nor, "Design and Fabrication of Silicon Nanowire based Sensor," Int. J. Electrochem. Sci, vol. 8, no. 9, pp. 1094610960, Aug. 2013.

[4] G. Yona, W. Dirks \& S. Rahman, "Comparing algorithms for clustering of expression data: how to assess gene clusters," Meth. Mol. Biol., vol. 541, pp. 479-509, Mar. 2009. https://doi.org/10.1007/978-1-59745-243-4_21

[5] A. Boulkroune, M. Tadjine, M. M'Saad \& M. Farza, "Fuzzy adaptive controller for MIMO nonlinear systems with known and unknown control direction," Fuzzy Sets Syst., vol. 161, no. 6, pp. 797-820, Mar. 2010. https://doi. org/10.1016/j.fss.2009.04.011

[6] Craig Venter Institute, GNN Genome News Network. http://www.genomenewsnetwork.org (accessed may. 26, 2010).

[7] C. Sanabria, O. Hernández \& A. K. Hay, "Estrategia de control para un inversor monofásico, con capacidad de conexión a un Bus AC utilizando el método de Droop," Rev. Col. Tecnol. Avan., vol. 2, no. 28, 39-47, May. 2017.

[8] J. L. Grosso. J. E. Forero, C. E. Cuadrado \& F. Otero, "Sistema de homogenización y control de viscosidad del combustóleo," CT\&F, vol. 1, no. 2, pp. 77-85, Ene. 1996.

[9] A. F. Muñoz, "Diseño, desarrollo e implementación de un dispositivo de pruebas de electrohilado (electrospinning) para aplicaciones en nanoinstrumentación," tesis pregrado, Fac. Ing. Mecat., UNAB, STD, Co, 2017.

[10] F. Vera, "Sistema electrónico de clonacion artificial de un sensor de viscosidad basado en hardware evolutivo," tesis pregrado, Fac. Ing. Electron., UDP, STD, Co, 2006.

[11] A. F. Muñoz, Sensorica e instrumentación, Mecánica de Alta precisión, Habana, Cuba: Pueblo y educación, 1997.

[12] N. Maneiro, "Algoritmos genéticos aplicados al problema cuadrático de asignación de facilidades," tesis pregrado, Dept. Inv. Ope, Esc. Ing. Ind., UC, VLN, Ve, 2002.

[13] J. Loyo, "Algoritmos Genéticos y Optimización en la Investigación de Operaciones. Caso: Problema de Optimización con Objetivos Múltiples Expresado como un Modelo de Programación No - Lineal por Metas," tesis pregrado, Dept. Comp., Fac. Ci. Tecnol., UC, VLC, Ve. 2002.

[14] H. Paba, "Implementación de sensores inteligentes utilizando redes neuronales aplicadas en procesos de refinación del petróleo," Msc tesis, UNAB, STD, Co, 2000.

[15] H. Boudouda, H. Seridi \& H. Akdag, "The Fuzzy Possibilistic C-Means Classifier," Asian J. Inf. Technol, vol. 4, no. 11, pp. 981-985, 2005. http://medwelljournals.com/ abstract/?doi=ajit.2005.981.985

[16] B. Rueda, "Nueva micro-nanobioinstrumentación inteligente en red de sensores nariz electrónica-lengua artificial-ojo espectrofotométrico para el monitoreo de la calidad del agua (fenoles) en línea de tuberías para vertimiento y en efluentes," Proyecto Semillero Instrumentación y Control, UNAB, STD, Co, 2017.

[17] J. D. Guerrero, "Algoritmos de procesamiento de imágenes y redes neuronales artificiales para el reconocimiento del lenguaje de signos colombiano (LSC)," Rev. Col. Tecnol. Avan., vol. 2, no. 26, pp. 1-8, May. 2016.

[18] M. Serrano, Nuevos sensores sismológicos basados en la nanotecnología, La Lisa, La Habana, Cu: Centro Nacional de Investigaciones Sismológicas, 2001.

[19] J. Gómez, A. Muñoz, O. Gualdrón, "Algoritmos y sistemas basados en patrones de células madre y clonación artificial para la automatización y control en una operación de ensamblaje en procesos de metalurgia," Rev, Col. Tecnol. Avan., vol. 1 no. 25, pp. 62-82, Oct. 2015.

[20] J. Sanabria, "Herramienta software para Implementar minería de datos: clusterización utilizando lógica difusa", Orinoquia, vol. 8. no. 1, pp. 15-23, Jun. 2017. 
Antonio Faustino Muñoz-Moner tiene Doctorado en Control y Automatización Industrial de la Universidad de Minas (San Petersburgo). Magister en Control Electrónica y Automatización Industrial de la Universidad de Acero y Aleaciones de Moscú (Rusia). Especialización en Automatización Industrial de la Universidad de Acero y Aleaciones de Moscú (Rusia). Especialista en docencia universitaria de la Universidad de La Habana (Cuba). Ingeniero Metalúrgico-Automatización de la Universidad de Acero y Aleaciones de Moscú (Rusia).

Aldo Pardo Garcia es Doctor en Ingeniería Eléctrica en la Universidad Técnica Nacional de Belarús (Bielorrusia). Doctor en Ingeniería Eléctrica en la Universidad de Camagüey (Cuba). Con estudios de posdoctorado en el CINVESTAV de Guadalajara (México) y posdoctorado en la Universidad Internacional de Florida (Estados Unidos). Ingeniero eléctrico (MSc, Esp.) de la Universidad Técnica Agraria Estatal de Belarús (Bielorrusia). https://orcid.org/0000-0003-2040-9420

Pedro Miguel Caicedo-Torres es Doctor en Ingeniería de la Universidad Autónoma de Bucaramanga (Colombia). Especialista en Gerencia en Recursos Energéticos de la Universidad Autónoma de Bucaramanga (Colombia). Ingeniero en Mecatrónica de la Universidad Autonoma de Bucaramanga (Colombia). 